\title{
The role of the MAP kinase-kinase protein StMKK1 in potato immunity to different pathogens
}

\author{
Xiaokang Chen ${ }^{1}$, Wenbin Wang ${ }^{1}$, Pingping Cai ${ }^{1}$, Ziwei Wang ${ }^{1}$, Tingting $\mathrm{Li}^{1}$ and Yu Du (1)
}

\begin{abstract}
Mitogen-activated protein kinase (MAPK) cascades play important roles in plant immunity. Previously, we reported that the potato StMKK1 protein negatively regulates Nicotiana benthamiana resistance to Phytophthora infestans. However, the functions of StMKK1 in potato immunity are unknown. To investigate the roles of StMKK1 in potato resistance to different pathogens, such as the potato late-blight pathogen $P$. infestans, the bacterial wilt pathogen Ralstonia solanacearum, and the gray-mold fungal pathogen Botrytis cinerea, we generated StMKK1 transgenic lines and investigated the response of potato transformants to destructive oomycete, bacterial, and fungal pathogens. The results showed that overexpression and silencing of StMKK1 do not alter plant growth and development. Interestingly, we found that StMKK1 negatively regulated potato resistance to the hemibiotrophic/biotrophic pathogens $P$. infestans and $R$. solanacearum, while it positively regulated potato resistance to the necrotrophic pathogen $B$. cinerea. Further investigation showed that overexpression of StMKK1 suppressed potato pathogen-associated molecular pattern (PAMP)-triggered immunity (PTI) and salicylic acid (SA)-related responses, while silencing of StMKK1 enhanced PTI and SA-related immune responses. Taken together, our results showed that StMKK1 plays dual roles in potato defense against different plant pathogens via negative regulation of PTI and SA-related signaling pathways.
\end{abstract}

\section{Introduction}

Potato, the world's third most important food crop (FAOSTAT 2013), is threatened by a group of major pathogens, Phytophthora infestans, Ralstonia solanacearum, and Botrytis cinerea. P. infestans, a hemibiotrophic pathogenic oomycete ${ }^{1}$, is the causal agent of potato late blight, which results in global costs of more than 6 billion dollars per year ${ }^{2}$. The biotrophic bacterial pathogen $R$. solanacearum causes one of the most notorious diseases of potato, known as potato bacterial wilt ${ }^{3}$. Potato gray mold is a common disease and is caused by the necrotrophic fungal pathogen $B$. cinerea ${ }^{4}$. These diseases lead to billions of dollars of potato production losses annually and pose serious threats to food security.

\footnotetext{
Correspondence: Tingting Li (tingting.li@nwafu.edu.cn) or Yu Du (yu.du@nwafu.edu.cn)

${ }^{1}$ College of Horticulture, Northwest A\&F University and Shaanxi Engineering Research Center for Vegetables, Yangling, Shaanxi 712100, China

These authors contributed equally: Xiaokang Chen, Wenbin Wang
}

To combat pathogens, plants have evolved two-layered plant immune systems ${ }^{5}$. The first layer relies on patternrecognition receptors (PRRs), which can percept pathogen-associated molecular patterns (PAMPs). The perception of PAMPs triggers a series of defense responses called PAMP-triggered immunity $(\mathrm{PTI})^{6}$. The second layer is mediated by plant-resistance $(R)$ proteins that can detect cognate pathogen effectors and subsequently activate a robust immune response called effector-triggered immunity (ETI $)^{7}$.

The mitogen-activated protein kinase (MAPK) cascade is an important pathway that transduces extracellular stimuli into intracellular responses ${ }^{8,9}$. MAPK cascades generally contain three kinase components, MAP kinase kinase kinase (MAPKKKs), MAP kinase kinase (MAPKKs), and MAPKs ${ }^{10}$. It has been shown that the activation of both PRR proteins and $\mathrm{R}$ proteins can induce MAPK cascades, which play central roles in signaling defense responses ${ }^{11-13}$. For example, MEKK1-MKK4/ MKK5-MPK3/MPK6, which can be activated by the

\section{(c) The Author(s) 2021}

(c) (i) Open Access This article is licensed under a Creative Commons Attribution 4.0 International License, which permits use, sharing, adaptation, distribution and reproduction cc) in any medium or format, as long as you give appropriate credit to the original author(s) and the source, provide a link to the Creative Commons license, and indicate if changes were made. The images or other third party material in this article are included in the article's Creative Commons license, unless indicated otherwise in a credit line to the material. If material is not included in the article's Creative Commons license and your intended use is not permitted by statutory regulation or exceeds the permitted use, you will need to obtain permission directly from the copyright holder. To view a copy of this license, visit http://creativecommons.org/licenses/by/4.0/. 
bacterial PAMP flg22, could induce WRKY transcription factor gene expression and positively regulate plant defense against bacterial and fungal pathogens ${ }^{14,15}$. In addition, another well-demonstrated MAPK cascade in Arabidopsis, consisting of MKK1/MKK2 and MPK4, negatively regulates plant immunity by regulating the expression of $P R$ genes and the accumulation of $\mathrm{H}_{2} \mathrm{O}_{2}{ }^{16}$. In addition, AtMKK3, which can enhance the expression of $P R$ genes, was shown to play a role in the defense against Pseudomonas syringae pv. tomato DC3000 ${ }^{17}$.

MKKs play dual roles in plant defense against different pathogens. For example, overexpression of GhMKK1 decreases $N$. benthamiana resistance to $R$. solanacearum $^{18}$, while ZmMKK1 positively regulates $N$. tabacum resistance to Pseudomonas solanacearum ${ }^{19}$. Although the role of several MKK proteins in plant immunity has already been studied in Arabidopsis, their role in the potato response to different classes of pathogens is still unknown. Several MKK proteins were reported to play a role in SA-related immune signaling ${ }^{20}$. For example, tomato SIMKK2 and SIMKK4 were shown to be involved in both JA- and SA-signaling pathways ${ }^{21}$. Constitutively active AtMKK2-EE was shown to reduce SA levels upon P. syringae infection ${ }^{22}$.

Previously, we showed that the potato StMKK1 protein is a host target of the $P$. infestans RXLR effector. Overexpression of StMKK1 in N. benthamiana promotes plant susceptibility to $P$. infestans, indicating that StMKK1 negatively regulates plant immunity to the late-blight pathogen. However, the functions of StMKK1 in potato to $P$. infestans and other pathogens remain unknown. In this study, to dissect the role of StMKK1 in potato resistance to different plant pathogens, we constructed StMKK1 transgenic potato and investigated the response of potato transformants to oomycete, fungal, and bacterial pathogens.

\section{Results and discussion}

\section{Phylogenetic analysis of StMKK1}

Arabidopsis encodes ten $M K K$ genes, and AtMKK1 and AtMKK2 are very similar to each other ${ }^{23}$. To determine whether StMKK1 is redundant in potato, we identified all MKK proteins in potato, tomato, and $N$. benthamiana and performed a phylogenetic analysis with Arabidopsis MKKs (Supplemental Fig. 1). Consistent with a previous study, four clades of MKKs were found in Arabidopsis and in these three Solanaceae plants. An ancient duplication event in clade A resulted in the formation of two distinct subclades of MKKs, MKK1/2, and MKK6. Additionally, several recent duplication events of $M K K$ genes were found within both $N$. benthamiana and Arabidopsis, while no recent duplication was found in Solanum, i.e., potato and tomato. Therefore, it is clear that only one $M K K 1$ gene is present in the potato genome. Close examination of the MKK1 proteins of Arabidopsis and potato and tomato revealed that StMKK1 has 11 conserved subdomains and a conserved phosphorylation motif (S/T-xxxxx-S/T) in the activation loop (Supplemental Fig. 2). These data suggest that there is only one typical MKK1 gene in potato.

\section{StMKK1 negatively regulates plant immunity to Phytophthora pathogens}

Previously, we showed that StMKK1 negatively regulates $N$. benthamiana resistance to $P$. infestans. To investigate the role of StMKK1 in potato immunity, we analyzed the expression patterns of StMKK1 upon $P$. infestans infection and SA treatment. The results showed that StMKK1 expression was induced at the early infection stages during $P$. infestans infection (Supplemental Fig. 3a). Since SA plays an essential role in plant immunity, we checked the expression patterns of StMKK1 after SA treatment, and the results showed that StMKK1 was also induced after SA treatment (Supplemental Fig. 3b). These results suggested that StMKK1 plays a role in plant defense responses against $P$. infestans and the SA-related signaling pathway. To further confirm this, we analyzed stable potato transformants overexpressing GFP-StMKK1 and silencing StMKK1 (StMKK1-RNAi). The expression levels of StMKK1 were significantly upregulated in the different overexpression (OE) transgenic plants and downregulated in the RNAi transgenic plants, as determined by qRT-PCR (Supplemental Fig. 4). The StMKK1 OE plants, as well as the StMKK1-silencing plants (RNAi lines we constructed previously) ${ }^{24}$, showed no morphologically distinct phenotypes compared to the wild-type plant Desiree (Fig. 1a, b).

To analyze the roles of StMKK1 in potato immunity, detached leaf assays were performed for StMKK1 OE and RNAi lines. The middle leaves of 5-week-old potato transformants were detached and inoculated with a $P$. infestans zoospore suspension. At 4 days after inoculation (dai), lesion development was photographed, and lesion diameters were measured ${ }^{25}$. The results showed that StMKK1 OE lines developed larger lesions (Fig. 1c-e), while the RNAi lines developed smaller lesions than the control (Fig. 1f-h). These results indicate that StMKK1 negatively regulates plant immunity to the late-blight disease pathogen $P$. infestans in potato, similar to our previous findings in N. benthamiana ${ }^{26}$.

To investigate whether StMKK1 plays similar roles in plant immunity to other oomycete pathogens, we transiently expressed GFP-StMKK1 and GFP-GUS in N. benthamiana leaves and inoculated the leaves with Phytophthora parasitica zoospores. Lesion diameters were measured at 3 dai, and the results showed that GFPStMKK1-expressing leaves developed significantly larger lesions than control leaves (Supplemental Fig. 5). Taken together, these results indicate that StMKK1 accelerates 
(a)

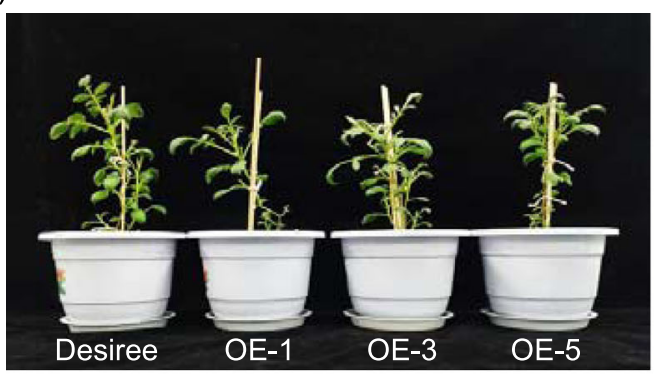

(c) Desiree
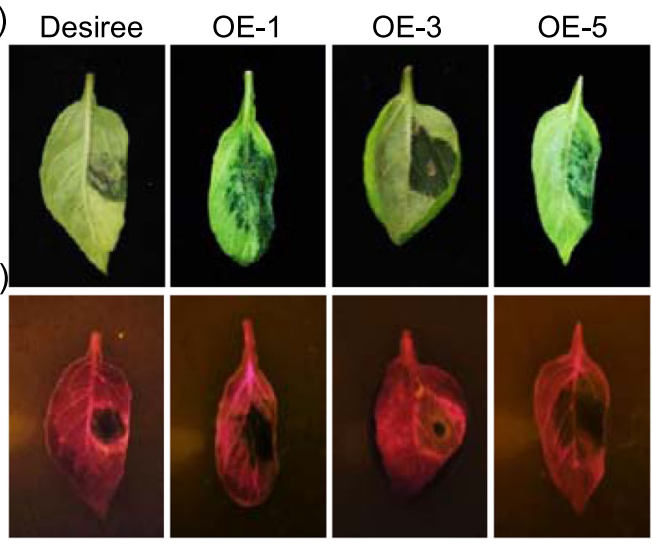

(e)

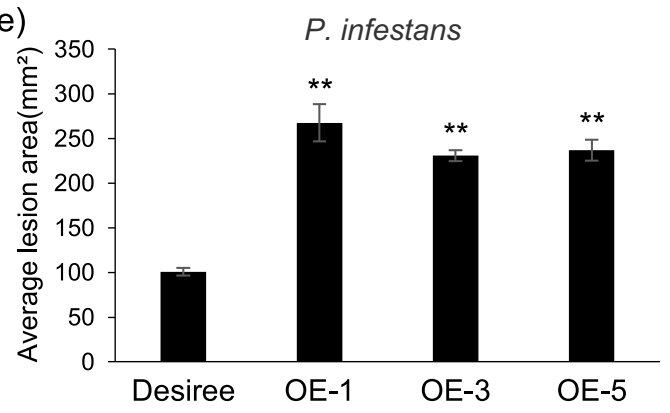

(b)

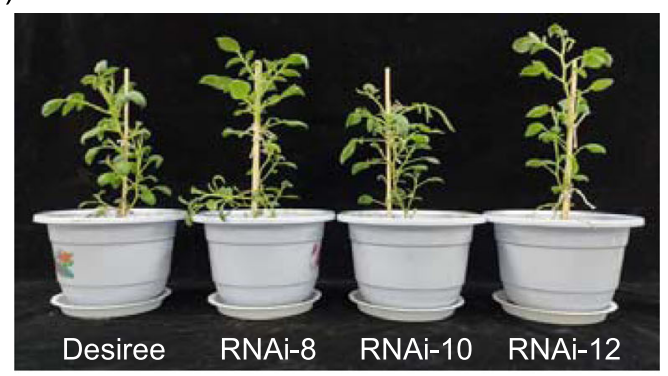

(f)
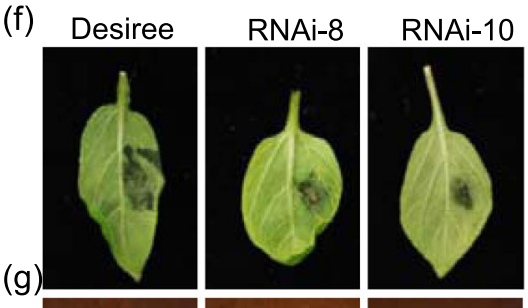

RNAi-12
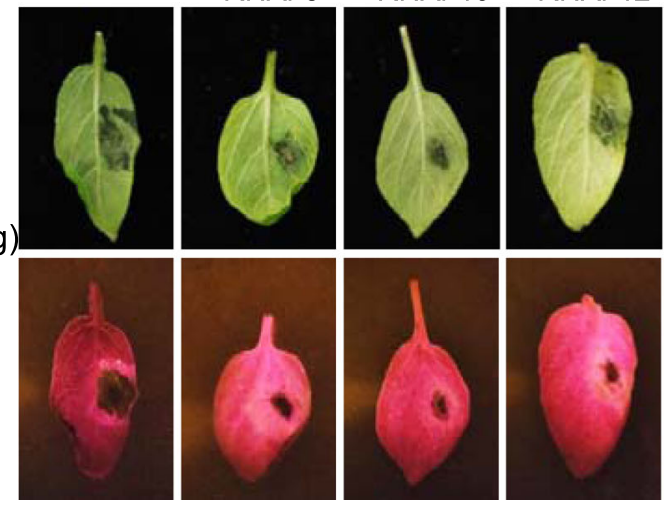

(h)

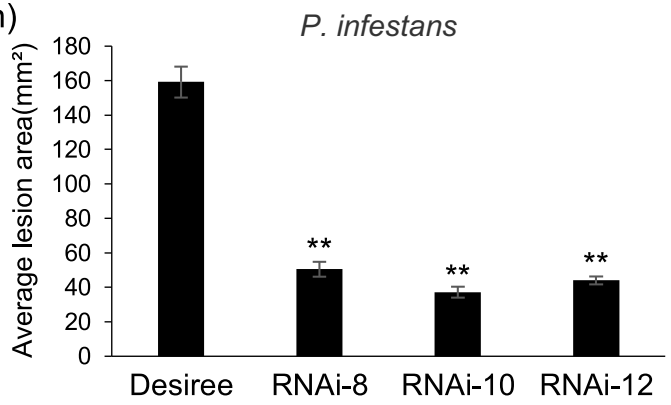

Fig. 1 StMKK1 negatively regulates potato resistance to $P$. infestans. The morphology of StMKK1 overexpression (a) and RNAi (b) transgenic potato plants. Representative images of Desiree- and StMKK1-overexpressing transgenic potato leaves with $P$. infestans lesions under natural light (c) and blue light (d). e Statistical analyses show that overexpression of StMKK1 in potato significantly increases $P$. infestans lesion areas compared to those in the wild-type Desiree. Representative leaf images of Desiree and StMKK1 RNAi transgenic potato leaves with $P$. infestans lesions under natural light (f) and blue light (g). $\mathbf{h}$ Statistical analyses show that silencing StMKK1 in potato significantly decreases $P$. infestans lesion areas compared with those in Desiree. All leaves were infected with zoospores of $P$. infestans isolate Pi14-3-GFP and photographed at 4 dai. Each inoculation test was repeated three times with similar results. In (e) and $\mathbf{( h )}$, error bars show the standard deviations from 16 replicates, and two-sided $t$-tests were used to assess significance: ${ }^{* *}, p<0.01$

the infection of Phytophthora pathogens and acts as a negative regulator of plant resistance against Phytophthora pathogens.

\section{StMKK1 negatively regulates potato resistance to the bacterial wilt pathogen Ralstonia solanacearum}

To test the role of StMKK1 in potato bacterial wilt disease, three independent transgenic lines, RNAi-8/10/
12, were grown in liquid MS medium for 2 weeks before transformation into distilled tap water comprising $1 \times 10^{8}$ $\mathrm{cfu} / \mathrm{mL} R$. solanacearum. The wilting symptoms were photographed, and the growth of bacteria was checked at 5 dai. As shown in Fig. 2, the control plants developed clear wilting symptoms, while StMKK1-silenced lines developed almost no symptoms. The quantification of bacterial growth confirmed our observation that the 
(a)
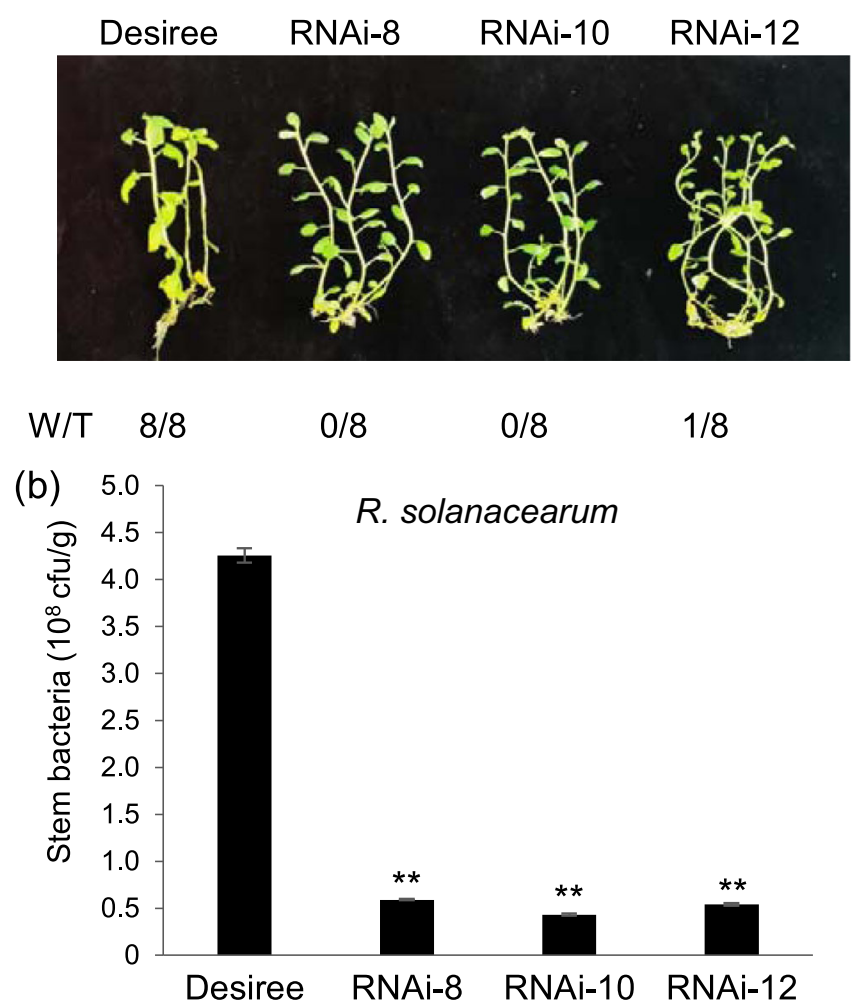

Fig. 2 Silencing of StMKK1 increases potato defense against Ralstonia solanacearum. a Representative images showing wilt symptoms in the wild-type Desiree and StMKK1 RNAi transgenic lines. W/T represents the number of wilting plants with respect to the total number of infected plants. b Statistical analysis of the amount of bacteria in plant stems showed significantly reduced bacterial colonization in StMKK1 RNAi lines compared with that in Desiree. Two-week-old potato plants were infected by the hydroponic infection method and photographed at 5 dai. The test was repeated three times with similar results. Error bars show the standard deviations from eight replicates. Two-sided $t$-tests were used to assess significance: **, $p<0.01$

StMKK1-silenced plants contained significantly fewer bacteria than the control plants. This result indicates that StMKK1 also negatively regulates plant immunity to the bacterial wilt pathogen $R$. solanacearum. Similarly, in cotton, it was reported that GhMKK1 negatively regulates plant resistance to $R$. solanacearum ${ }^{18}$.

\section{StMKK1 enhances potato resistance to the fungal pathogen Botrytis cinerea}

To test the role of StMKK1 in response to the necrotrophic fungal pathogen $B$. cinerea, two independent transgenic lines of StMKK1 OE-3/5 and RNAi-8/10 were used for infection. Middle leaves of 5-week-old plants were harvested and inoculated with $B$. cinerea. Lesion development was photographed, and the lesion diameters were measured at 2 dai. The results show that the StMKK1 OE-3/5 lines developed smaller lesions (Fig. $3 \mathrm{a}-\mathrm{c}$ ), while the RNAi-8/10-silenced lines developed larger lesions than the control plants (Fig. 3d-f). These results showed that StMKK1 positively regulates potato resistance to the necrotrophic plant pathogen $B$. cinerea.
This is in contrast to the hemibiotrophic and biotrophic pathogens $P$. infestans and $R$. solanacearum, for which StMKK1 negatively regulates potato immunity.

Many genes are reported to play dual roles in plant immunity; on the one hand, these genes contribute to plant susceptibility to biotrophic pathogens, and on the other hand, they promote plant resistance to necrotrophic pathogens ${ }^{27,28}$. There are two possibilities for this phenomenon. First, plant PTI responses result in the activation of reactive oxygen species (ROS) bursts and immunerelated gene expression, which in some cases, leads to plant cell death that is unfavorable for biotrophic pathogens, as they require a biotrophic environment for disease development. However, necrotrophic plant pathogens use plant cell death responses to kill the host cell for proliferation ${ }^{29}$; thus, plants express an opposite response to these pathogens. Second, different plant hormones respond differently to biotrophic/hemibiotrophic or necrotrophic pathogens. For example, SA positively regulates a large portion of the plant immune response to biotrophic and hemibiotrophic plant pathogens ${ }^{30,31}$, while 
(a)

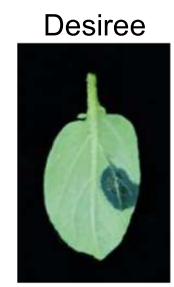

(b)

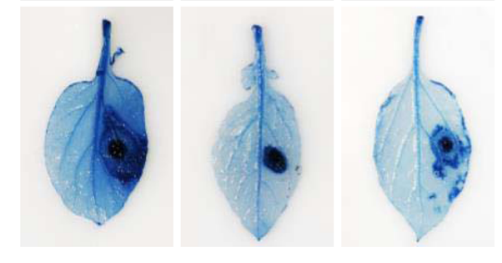

(c)

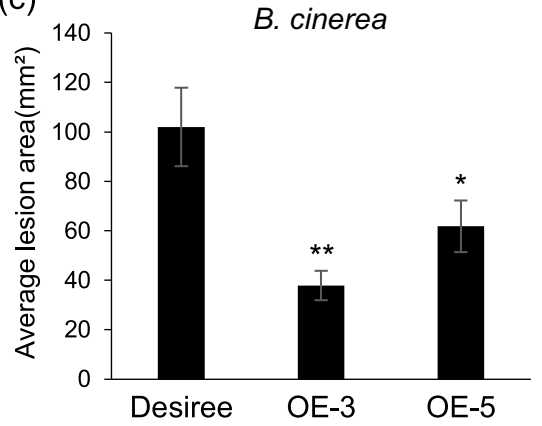

(d)

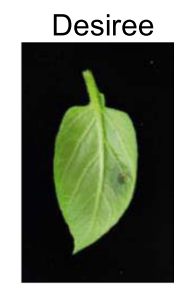

RNAi-8

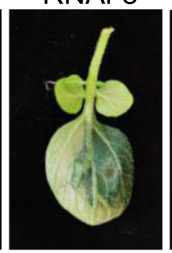

RNAi-10

(e)

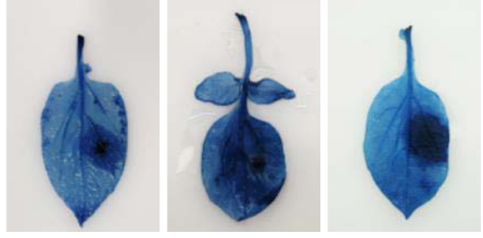

(f)

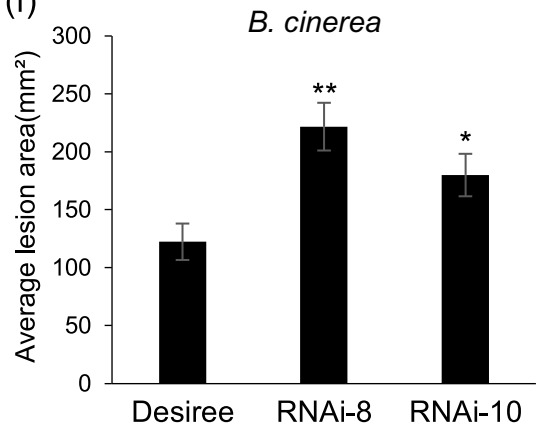

Fig. 3 StMKK1 positively regulates potato resistance to Botrytis cinerea. Representative leaf images of StMKK1 overexpression transgenic lines before (a) and after (b) trypan blue staining showing lesions of B. cinerea at 2 dai. c Bar graph showing that overexpression of StMKK1 in potato significantly reduces $B$. cinerea lesion areas compared to that in the wild-type Desiree. Representative leaf images of StMKK1 RNAi transgenic lines before (d) and after (e) trypan blue staining showing B. cinerea lesions developed at 2 dai. $\mathbf{f}$ Bar graph showing that silencing of StMKK1 in potato significantly increases $B$. cinerea lesion areas compared to that in Desiree. All leaves were infected with B. cinerea B05.10. Each inoculation test was repeated three times with similar results. In (c) and (f), error bars show the standard deviations from 16 replicates, two-sided t-tests were used to assess significance: ${ }^{*}, p<0.05, * *, p<0.01$

it negatively regulates plant immunity to necrotrophic pathogens ${ }^{32}$.

\section{StMKK1 inhibits potato PTI responses}

To further investigate the mechanism by which StMKK1 regulates plant immunity, we checked the PTI responses in StMKK1 OE-1/3/5 and RNAi-8/10/12 lines. The transgenic plants were infiltrated with flg22, and PTIrelated gene expression and ROS bursts were detected. As shown in Fig. 4a, c, the gene expression of StFRK1 and StWRKY7 was reduced and the flg22-triggered ROS burst was suppressed in StMKK1 OE-1/3/5 plants. However, the RNAi-8/10/12 lines showed enhanced expression of PTI-related genes and induction of an ROS burst (Fig. 4b, d). These results indicate that StMKK1 negatively regulates plant PTI responses.

\section{StMKK1 negatively regulates SA-related immunity}

In Arabidopsis, the Atmkk1/2 mutant shows enhanced salicylic acid (SA)-related disease resistance ${ }^{33}$. To reveal the role of potato StMKK1 in the SA-related defense response, we analyzed the effect of StMKK1 on SAresponsive gene expression. StMKK1 was transiently expressed in $N$. benthamiana leaves for 2 days before harvest to assess SA-responsive gene expression. The results showed that the expression of $N b P R 1, N b P R 2$, NbPR5, and NbICS1 was significantly repressed in StMKK1-expressing leaves compared with that in the GFP-GUS control (Fig. 5a). Moreover, in NbMKK1/2silenced $N$. benthamiana plants, the expression of $N b P R 1$, NbPR2, NbPR5, and NbICS1 was induced significantly compared to that in control plants (Fig. 5b), which again indicates its role in the regulation of SA-related plant immunity. Consistently, qRT-PCR data in potato indicated that the expression of StPR1, StPR2, StPR5, and StICS1 was repressed in StMKK1 overexpression lines (Fig. 5c) and induced in StMKK1 RNAi lines (Fig. 5d).

In Arabidopsis, the MEKK1-MKK1/2-MPK4 cascade was monitored by the NB-LRR protein SUMM2 ${ }^{33,34}$, and mutation of MKK1/2 resulted in the activation of SUMM2 and subsequently led to the activation of SArelated immunity. Consequently, Arabidopsis mkk1/2 and 

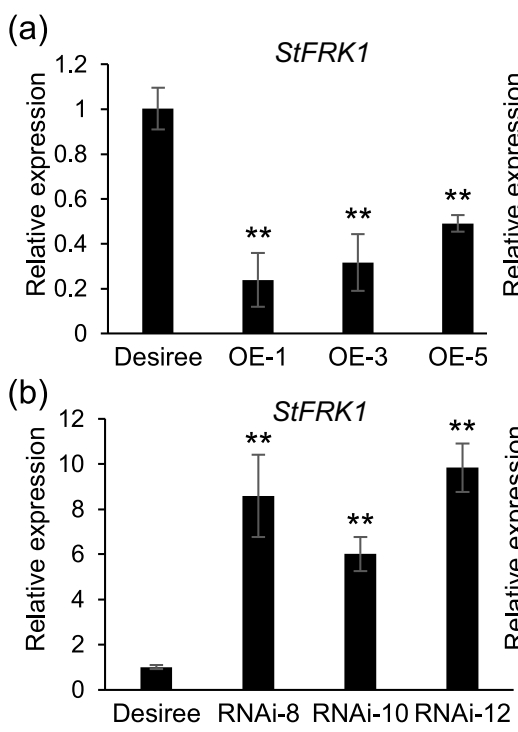

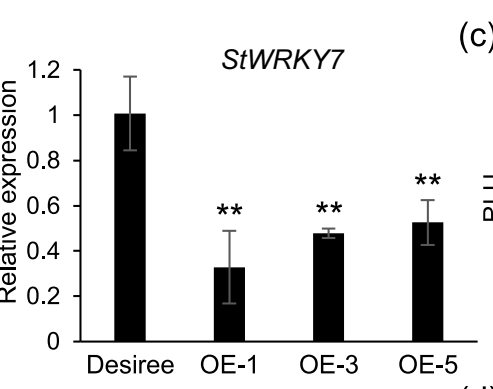

StWRKY7

(c)

(d)
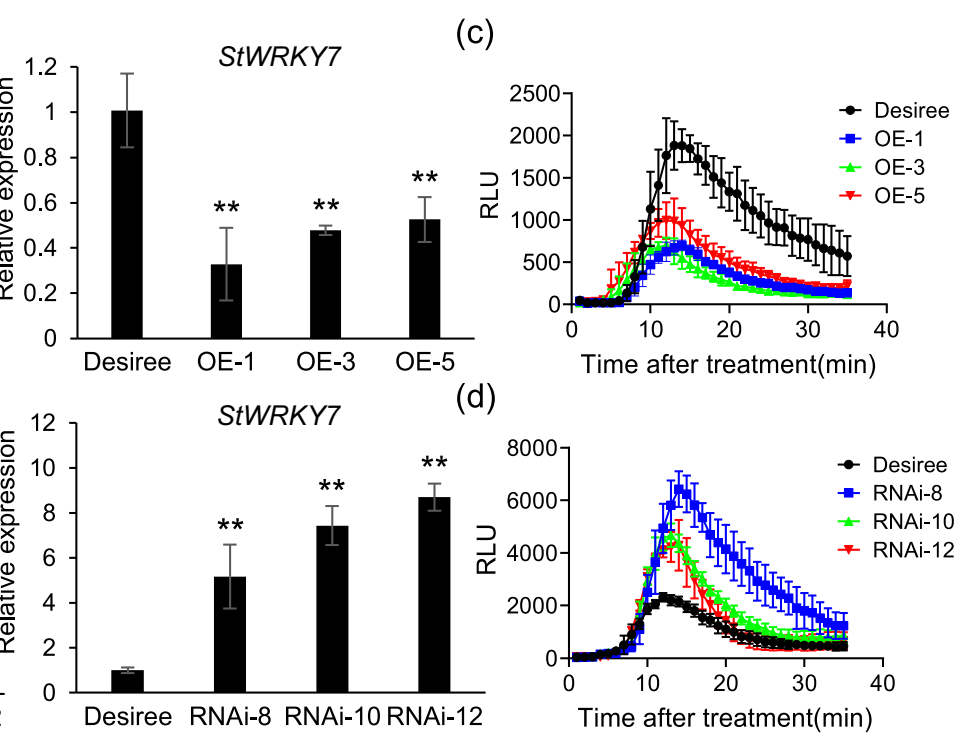

Fig. 4 StMKK1 suppresses plant PTI responses. QRT-PCR analysis showing the expression of the PTI marker genes StFRK1 and StWRKY7 in StMKK1 overexpression (a) and StMKK1 RNAi transgenic potato lines (b). Total RNA was extracted from $40 \mu \mathrm{M}$ flg22-treated leaves of the corresponding plants. StActin was used as a reference gene in potato. StFRK1 and StWRKY7 gene expression levels in Desiree were set to 1. One-sided t-tests were used to assess significance: ${ }^{*}, p<0.01$. Flg22-induced ROS production was measured in StMKK1 overexpression (c) and StMKK1 RNAi transgenic potato lines (d). The wild-type Desiree was used as a control. Ten microliters of flg22 was used before the measurement of ROS production. RLU represents relative luminescence units. Data were analyzed by GraphPad Prism 6.0. Each experiment was repeated three times with similar results. Error bars show the standard deviations from three technical replicates
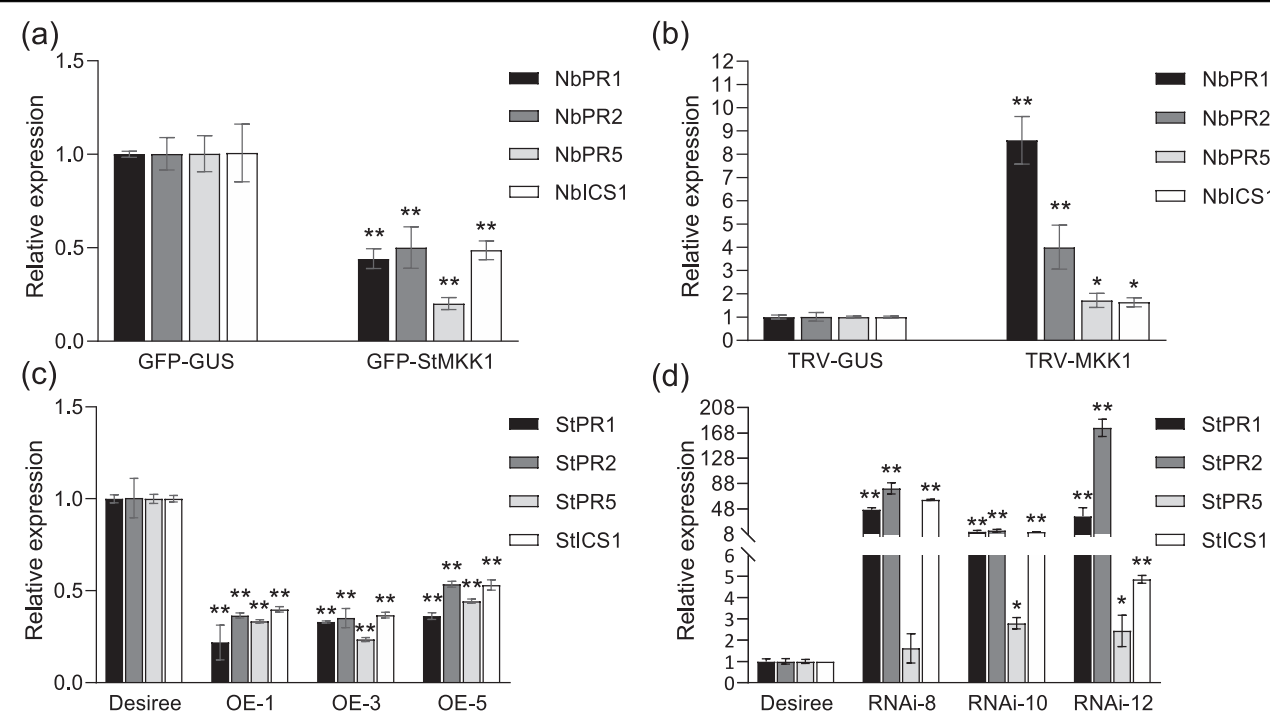

Fig. 5 StMKK1 negatively regulates the expression of SA marker genes. The relative expression of SA marker genes in N. benthamiana expressing GFPGUS and GFP-STMKK1 (a), TRV-GUS and TRV-MKK1 (b), and in STMKK1 overexpression (c) and StMKK1 RNAi transgenic potato lines (d). Total RNA was extracted from uninfected leaves of the corresponding plants. SA marker genes PR1, PR2, PR5, and ICS1 were examined. NbActin and StActin were used as reference genes in $N$. benthamiana and potato, respectively ${ }^{45}$. The expression levels of SA marker genes in GFP-GUS, TRV-GUS, and Desiree were set to 1 , respectively. One-sided $t$-tests were used to assess significance: ${ }^{*}, p<0.05,{ }^{* *}, p<0.01$. Error bars represent the standard deviations from three biological replicates

mpk4 mutants showed lesion mimic phenotypes ${ }^{33,35}$. However, in both $N$. benthamiana and potato, silencing StMKK1 did not alter plant growth; thus, we hypothesized that solanaceous plants may not have a functional SUMM2 gene in their genome. It is likely that StMKK1 uses other mechanisms to repress SA-related immunity. 
Taken together, our results and previous studies have shown that StMKK1 negatively regulates the PTI response and SA-dependent immunity. It was reported that SA activates both PTI and ETI responses, which subsequently activate MAPK cascade signaling. Thus, it is not surprising to find that SA activated StMKK1 gene expression (Supplemental Fig. 3). Since plant MAPK cascades regulate complicated cellular processes, including plant immunity, the magnitude of MAPK cascade activation must be accurately regulated to avoid overactivation of plant immunity, which likely leads to the inhibition of plant development. For example, it was reported that the overexpression of $N$. benthamiana constitutively activated NbMEK2 ${ }^{\text {DD36 }}$, and Arabidopsis AtMKK7 and AtMKK9 induced plant cell death ${ }^{37}$. Thus, to repress the overactivation of MAPK cascades, plants have evolved protein phosphatases to dephosphorylate the MAPK cascade ${ }^{38,39}$. In our study, we found that StMKK1 negatively regulates the PTI response and SA-dependent immunity, and it is likely that plants employ StMKK1 to avoid the overactivation of immunity.

\section{Conclusion}

In summary, we showed that overexpression of StMKK1 in potato inhibits plant resistance to $P$. infestans and $R$. solanacearum while enhancing plant resistance to $B$. cinerea, likely by suppressing plant PTI and SA-related immunity. Silencing MKK1 enhances plant resistance to colonization by the hemibiotrophic P. infestans and biotrophic $R$. solanacearum, but reduces plant resistance to the necrotrophic $B$. cinerea. Our results showed that StMKK1 plays different roles in potato resistance against biotrophic and necrotrophic pathogens by negatively regulating PTI and SA-related signaling pathways.

\section{Materials and methods Agroinfiltration}

GFP-StMKK1 and StMKK1-pART27-RNAi plasmids were constructed as described previously ${ }^{24,26}$. Agrobacterium strain C58C1 carrying GFP-StMKK1 or GFPGUS plasmids was cultured in liquid LB medium with appropriate antibiotics at $28^{\circ} \mathrm{C}$. Two days later, the Agrobacterium cells were resuspended in infiltration buffer (10 mM 2-(N-morpholino) ethanesulfonic acid (MES), $10 \mathrm{mM} \mathrm{MgCl}_{2}$, and $200 \mathrm{mM}$ acetosyringone, $\mathrm{pH}$ 5.6) to an OD600 of 0.3 and kept at room temperature for $1 \mathrm{~h}$ before infiltration into $N$. benthamiana leaves.

\section{Potato transformation}

The StMKK1-RNAi lines were described previously ${ }^{24}$. Agrobacterium tumefaciens-harboring pART27-StMKK1 was transformed into potato cv Desiree by stem segment transformation as described previously ${ }^{40}$. The rooted transformants grown on MS medium supplemented with vitamins, $100 \mathrm{mg} / \mathrm{L}$ kanamycin, and $30 \mathrm{~g} / \mathrm{L}$ sucrose were transferred to a new MS medium without kanamycin at $23^{\circ} \mathrm{C}$ with a $16 / 8 \mathrm{~h}$ day/night cycle. Three weeks later, the transformants were transferred to plastic pots containing potting soil in a climate chamber at $25^{\circ} \mathrm{C}$ with a $16 / 8 \mathrm{~h}$ day/night cycle. For both overexpression and RNAi constructs, more than five independent transformants were obtained and confirmed by PCR with the forward primer of the $35 \mathrm{~S}$ promoter and the gene-specific reverse primer of StMKK1 (Supplemental Table 1). For each transformant, more than three biological replicates were grown for further investigations.

\section{Pathogen strains and growth conditions}

$P$. infestans isolate 14-3-GFP was grown on rye and sucrose agar (RSA) plates at $18^{\circ} \mathrm{C}$ in the dark for approximately 2 weeks before zoospores were collected. 14-3-GFP is a GFP-expressing transformant of P. infestans H30P02 and was shown to reach a 100\% infection efficiency on Desiree in a previous study ${ }^{25}$. P. parasitica was grown on $5 \%$ carrot juice agar $(\mathrm{CA})$ plates at $23^{\circ} \mathrm{C}$ in the dark for 4-5 days, and the zoospores were prepared as described previously ${ }^{41}$. Botrytis cinerea strain B05.10 was grown on potato dextrose agar (PDA) plates in the dark at $23^{\circ} \mathrm{C}$ for 3-4 days before spores were collected. The $R$. solanacearum strain GMI1000 was grown in liquid LB medium overnight at $28^{\circ} \mathrm{C}$ in a shaker.

\section{Plant growth condition and pathogen infection assays}

Potato and $N$. benthamiana plants were grown in a climate chamber with a $16 / 8 \mathrm{~h}$ day/night cycle at $25^{\circ} \mathrm{C}$. Four- to five-week-old N. benthamiana plants and 5week-old potato plants were used for infection assays. Zoospores from $P$. infestans isolate 14-3-GFP were collected as described previously ${ }^{26}$. Detached leaf assays were performed by inoculating $10-\mu \mathrm{L}$ zoospore suspensions containing 1000 zoospores onto one potato leaflet. The inoculated leaves were kept in moisture in the dark at $18^{\circ} \mathrm{C}$ and the lesion diameters were measured at 4 dai. For $B$. cinerea inoculation, the spore suspension was prepared as described previously ${ }^{42}$, and for each leaf, a $2-\mu \mathrm{L}$ spore suspension that contained approximately 2000 spores was used. The inoculated leaves were kept at room temperature, and at 2 dai, the lesion diameters were measured. For $R$. solanacearum infection, $R$. solanacearum overnight cultures were collected and washed two times before dilution with distilled tap water to an OD600 of 0.1. Twoweek-old potato plants were inoculated with $R$. solanacearum suspensions using the method described previously $^{43}$. Wilting symptoms were observed at 5 dai, and the number of bacteria in the aerial parts of the infected plants (cfu/fresh weight) was counted as described previously $^{43}$. For Phytophthora parasitica infection, the GFPtagged strain Pp1121 was used. P. parasitica was cultured, 
and infection assays were performed as described previously $^{41}$, and the lesion diameters were measured at 3 dai.

\section{Quantitative real-time (qRT)-PCR}

Total RNA was isolated from transgenic potato lines using TRIzol reagent (Invitrogen). First-stranded cDNAs were synthesized, and qRT-PCR was performed as described $^{26}$ using the appropriate primer pairs shown in Supplemental Table 1. StMKK1 expression levels in different transgenic lines were quantified using the $2^{\Delta \Delta} \mathrm{Ct}$ method, and the potato gene Actin was used for normalization. Additionally, the expression of the N. benthamiana gene Actin was used for normalization.

\section{Flg22 treatments, ROS production analysis, and salicylic acid treatments}

The $10 \mu \mathrm{M}$ flg22-treated leaves were subsequently used for ROS production analysis as described previously ${ }^{44}$. To analyze the transcript level of StMKK1 under SA treatment, SA was dissolved in ethanol, and $10 \mathrm{mM}$ SA solution was sprayed onto 2-month-old potato leaves. Leaves were harvested at 1, 3, 6, 12, and $24 \mathrm{~h}$ after SA spraying for RNA extraction.

\section{Western blotting}

Samples expressing GFP-StMKK1 and GFP-GUS were extracted using lysis buffer as described previously ${ }^{26}$. Sodium dodecyl sulfate-polyacrylamide gel electrophoresis (SDS-PAGE) was performed to detect the proteins. Antibody (anti-GFP, goat anti-rabbit) was used according to descriptions given in the manual.

\section{Accession numbers}

Accession numbers of StMKK1 are as follows: Sotub12g010200.1.1. NbMKK1/2: Niben101Scf02790g03 012.1, Niben101Scf13387g00027.1, Niben101Scf10103g 03014.1, Niben101Scf00611g07010.1.

\begin{abstract}
Acknowledgements
We thank the Life Science Research Core Services and State Key Laboratory of Crop Stress Biology for Arid Areas (Northwest A\&F University, Yangling, China) for experimental assistance. This work was financed by the National Natural Science Foundation of China (31701770, 32072401), the China Postdoctoral Science Foundation (2019T120956 and 2016M600818), the Postdoctoral Science Foundation of Shaanxi Province (2017BSHYDZZ63), and the Northwest A\&F University Scientific Research Fund for Advanced Talents (2452018028 and 2452017069).
\end{abstract}

\section{Author contributions}

Y.D. and X.C. designed the research. X.C., W.W., P.C., and Z.W. performed the experiments. X.C., W.W., and Y.D. analyzed the data, and T.L. performed phylogenetic analysis of MKK1. Y.D., T.L., and X.C. wrote the paper. All authors reviewed the paper.

\section{Conflict of interest}

The authors declare no conflicts of interest.
Supplementary information The online version contains supplementary material available at https://doi.org/10.1038/s41438-021-00556-5.

Received: 15 November 2020 Revised: 18 February 2021 Accepted: 14 March 2021

Published online: 01 June 2021

\section{References}

1. Govers, F., Drenth, A. \& Pieterse, C. M. J. in Plant Relationships Part B (eds Carroll, G. C. \& Tudzynski, P.) 17-36 (Springer Berlin Heidelberg, 1997).

2. Haverkort, A. J. et al. Societal costs of late blight in potato and prospects of durable resistance through cisgenic modification. Potato Res. 51, 47-57 (2008).

3. Genin, S. Molecular traits controlling host range and adaptation to plants in Ralstonia solanacearum. N. Phytologist 187, 920-928 (2010).

4. van Kan, J. A. L. Licensed to kill: the lifestyle of a necrotrophic plant pathogen. Trends Plant Sci. 11, 247-253 (2006).

5. Jones, J. D. G. \& Dangl, J. L. The plant immune system. Nature 444, 323-329 (2006).

6. Chisholm, S. T., Coaker, G., Day, B. \& Staskawicz, B. J. Host-microbe interactions: shaping the evolution of the plant immune response. Cell 124, 803-814 (2006).

7. Cui, H. T., Tsuda, K. \& Parker, J. E. Effector-triggered immunity: from pathogen perception to robust defense. Annu. Rev. Plant Biol. 66, 487-511 (2015).

8. Davis, R. J. Signal transduction by the JNK group of MAP kinases. Cell 103, 239-252 (2000).

9. Chang, L. F. \& Karin, M. Mammalian MAP kinase signaling cascades. Nature 410, 37-40 (2001).

10. Jonak, C., Okresz, L., Bogre, L. \& Hirt, H. Complexity, cross talk and integration of plant MAP kinase signaling. Curr. Opin. Plant Biol. 5, 415-424 (2002).

11. Rodriguez, M. C. S., Petersen, M. \& Mundy, J. Mitogen-activated protein kinase signaling in plants. Annu. Rev. Plant Biol. 61, 621-649 (2010).

12. Pitzschke, A., Schikora, A. \& Hirt, H. MAPK cascade signaling networks in plant defence. Curr. Opin. Plant Biol. 12, 421-426 (2009).

13. Pedley, K. F. \& Martin, G. B. Role of mitogen-activated protein kinases in plant immunity. Curr. Opin. Plant Biol. 8, 541-547 (2005).

14. Asai, T. et al. MAP kinase signaling cascade in Arabidopsis innate immunity. Nature 415, 977-983 (2002)

15. Mao, G. H. et al. Phosphorylation of a WRKY transcription factor by two pathogen-responsive MAPKs drives phytoalexin biosynthesis in Arabidopsis. Plant Cell 23, 1639-1653 (2011).

16. Gao, M. H. et al. MEKK1, MKK1/MKK2 and MPK4 function together in a mitogen-activated protein kinase cascade to regulate innate immunity in plants. Cell Res. 18, 1190-1198 (2008).

17. Doczi, R. et al. The Arabidopsis mitogen-activated protein kinase kinase MKK3 is upstream of group C mitogen-activated protein kinases and participates in pathogen signaling. Plant Cell 19, 3266-3279 (2007).

18. Lu, W., Chu, X., Li, Y., Wang, C. \& Guo, X. Cotton GhMKK1 induces the tolerance of salt and drought stress, and mediates defence responses to pathogen infection in transgenic Nicotiana benthamiana. PLoS One 8, e68503 (2013).

19. Cai, G. et al. ZmMKK1, a novel group A mitogen-activated protein kinase kinase gene in maize, conferred chilling stress tolerance and was involved in pathogen defense in transgenic tobacco. Plant Sci. 214, 57-73 (2014).

20. Jagodzik, P., Tajdel-Zielinska, M., Ciesla, A., Marczak, M. \& Ludwikow, A. Mitogen-activated protein kinase cascades in plant hormone signaling. Front. Plant Sci. 9, 1387 (2018)

21. Li, X. et al. Tomato SIMKK2 and SIMKK4 contribute to disease resistance against Botrytis cinerea. BMC Plant Biol. 14, 166 (2014).

22. Brader, G., Djamei, A., Teige, M., Palva, E. T. \& Hirt, H. The MAP kinase kinase MKK2 affects disease resistance in Arabidopsis. Mol. Plant-Microbe Interact. 20, 589-596 (2007).

23. Hamel, L.-P. et al. Ancient signals: comparative genomics of plant MAPK and MAPKK gene families. Trends Plant Sci. 11, 192-198 (2006).

24. Chen, X., Li, F., Wang, W., Shan, W. \& Du, Y. Construction of RNA interference vector to silence StMKK1 in potato and development of StMKK1-silenced transgenic potato lines. Acta Agriculturae Boreal.-occidentalis Sin. 30, 1822-1830 (2020).

25. Bouwmeester, $K$. et al. The Arabidopsis lectin receptor kinase LecRK-1.9 enhances resistance to Phytophthora infestans in solanaceous plants. Plant Biotechnol. J. 12, 10-16 (2014). 
26. Du, Y. et al. Phytophthora infestans RXLR effector PITG20303 targets a potato MKK1 protein to suppress plant immunity. N. Phytologist 229, 501-515 (2021)

27. Wang, Y., Bouwmeester, K., Beseh, P., Shan, W. \& Govers, F. Phenotypic analyses of Arabidopsis T-DNA insertion lines and expression profiling reveal that multiple L-type lectin receptor kinases are involved in plant immunity. Mol. Plant-Microbe Interact. 27, 1390-1402 (2014).

28. Du, Y., Overdijk, E. J. R., Berg, J. A., Govers, F. \& Bouwmeester, K. Solanaceous exocyst subunits are involved in immunity to diverse plant pathogens. J. Exp. Botany 69, 655-666 (2018).

29. Mengiste, T. Plant immunity to necrotrophs. Annu. Rev. Phytopathol. 50, 267-294 (2012)

30. Pan, Q. et al. RTP1 encodes a novel endoplasmic reticulum (ER)-localized protein in Arabidopsis and negatively regulates resistance against biotrophic pathogens. N. Phytologist 209, 1641-1654 (2016).

31. Cui, $H$. et al. Pseudomonas syringae effector protein AvrB perturbs Arabidopsis hormone signaling by activating MAP kinase 4. Cell Host Microbe 7, 164-175 (2010).

32. Spoel, S. H., Johnson, J. S. \& Dong, X. Regulation of tradeoffs between plant defenses against pathogens with different lifestyles. Proc. Natl Acad. Sci. 104, 18842-18847 (2007).

33. Zhang, Z. et al. Disruption of PAMP-induced MAP kinase cascade by a Pseudomonas syringae effector activates plant immunity mediated by the NB-LRR protein SUMM2. Cell Host Microbe 11, 253-263 (2012).

34. Zhang, Z. et al. The NLR protein SUMM2 senses the disruption of an immune signaling MAP kinase cascade via CRCK3. EMBO Rep. 18, 292-302 (2016).

35. Kong, Q. et al. The MEKK1-MKK1/MKK2-MPK4 kinase cascade negatively regulates immunity mediated by a mitogen-activated protein kinase kinase kinase in Arabidopsis. Plant Cell 24, 2225-2236 (2012).
36. Yang, K. Y., Liu, Y. \& Zhang, S. Activation of a mitogen-activated protein kinase pathway is involved in disease resistance in tobacco. Proc. Natl Acad. Sci. 98, 741-746 (2001)

37. Popescu, S. C. et al. MAPK target networks in Arabidopsis thaliana revealed using functional protein microarrays. Genes Dev. 23, 80-92 (2008).

38. Jiang, L., Chen, Y., Luo, L. \& Peck, S. C. Central roles and regulatory mechanisms of dual-specificity MAPK phosphatases in developmental and stress signaling. Front. Plant Sci. 9, 1697 (2018).

39. Liu, Y., Shepherd, E. G. \& Nelin, L. D. MAPK phosphatases-regulating the immune response. Nat. Rev. Immunol. 7, 202-212 (2007).

40. Sun, $\mathrm{K}$. et al. Silencing of six susceptibility genes results in potato late blight resistance. Transgenic Res. 25, 731-742 (2016).

41. Fan, G. et al. A Phytophthora capsici RXLR effector targets and inhibits a plant PPlase to suppress endoplasmic reticulum-mediated immunity. Mol. Plant 11, 1067-1083 (2018).

42. Zhang, L. \& van Kan, J. A. L. Botrytis cinerea mutants deficient in D-galacturonic acid catabolism have a perturbed virulence on Nicotiana benthamiana and Arabidopsis, but not on tomato. Mol. Plant Pathol. 14, 19-29 (2012).

43. Wang, $\mathrm{H}$. et al. A quick and efficient hydroponic potato infection method for evaluating potato resistance and Ralstonia solanacearum virulence. Plant Methods 15, 145 (2019).

44. Li, L. et al. Activation-dependent destruction of a co-receptor by a Pseudomonas syringae effector dampens plant immunity. Cell Host Microbe $\mathbf{2 0}$ 504-514 (2016).

45. Zhou, $X$. et al. StMYB44 negatively regulates phosphate transport by suppressing expression of PHOSPHATE1 in potato. J. Exp. Botany 68, 1265-1281 (2017). 